\title{
Papers
}

\section{Non-absorbable disaccharides for hepatic encephalopathy: systematic review of randomised trials}

\author{
Bodil Als-Nielsen, Lise L Gluud, Christian Gluud
}

\begin{abstract}
Objective To assess the effects of non-absorbable disaccharides (lactulose and lactitol) in patients with hepatic encephalopathy. Data sources Cochrane Hepato-Biliary Group controlled trials register, Cochrane Library, Medline, and Embase until March 2003; reference lists of relevant articles; authors and pharmaceutical companies.

Review methods Randomised trials that compared non-absorbable disaccharides with placebo, no intervention, or antibiotics for hepatic encephalopathy were included. The primary outcome measures were no improvement of hepatic encephalopathy and all cause mortality.

Results 22 trials were included. Compared with placebo or no intervention, non-absorbable disaccharides seemed to reduce the risk of no improvement in patients with hepatic

encephalopathy (relative risk $0.62,95 \%$ confidence interval 0.46 to 0.84 , six trials). However, high quality trials found no significant effect $(0.92,0.42$ to 2.04 , two trials). Compared with placebo or no intervention, non-absorbable disaccharides had no significant effect on mortality $(0.41,0.02$ to 8.68 , four trials). Non-absorbable disaccharides were inferior to antibiotics in reducing the risk of no improvement (1.24, 1.02 to $1.50,10$ trials) and lowering blood ammonia concentration (weighted mean difference $2.35 \mu \mathrm{mol} / \mathrm{l}, 0.06 \mu \mathrm{mol} / \mathrm{l}$ to $13.45 \mu \mathrm{mol} / \mathrm{l}, 10$ trials). There was no significant difference in mortality $(0.90$, 0.48 to 1.67 , five trials).

Conclusions There is insufficient evidence to support or refute the use of non-absorbable disaccharides for hepatic encephalopathy. Antibiotics were superior to non-absorbable disaccharides in improving hepatic encephalopathy, but it is unclear whether this difference is clinically important.

Non-absorbable disaccharides should not serve as comparator in randomised trials on hepatic encephalopathy.
\end{abstract}

\section{Introduction}

Hepatic encephalopathy is a complex neuropsychiatric syndrome, which may complicate acute or chronic liver failure. ${ }^{1}$ It is characterised by changes in mental state including a wide range of neuropsychiatric symptoms ranging from minor signs of altered brain function to deep coma. ${ }^{2}$

Treatment of hepatic encephalopathy aims at reducing the production and absorption of ammonia, which is involved in the pathogenesis. ${ }^{3}$ As colonic bacteria are the primary source of ammonia, treatment initially consisted of poorly absorbed antibiotics, especially neomycin. ${ }^{56}$ This treatment was implemented without appropriate scientific documentation. Lactulose was introduced as a safer alternative. ${ }^{3}$ On the basis of two small trials, ${ }^{56}$ lactulose was considered to be as effective as neomycin. Subsequent trials and meta-analyses concluded that lactitol and lactulose were equally effective. ${ }^{7-10}$ Since the 1980 s, nonabsorbable disaccharides (lactulose and lactitol) have been considered as the standard treatment for hepatic encephalopathy. ${ }^{11}{ }^{12}$ Recent guidelines state that lactulose is the first line pharmacological treatment for hepatic encephalopathy. ${ }^{12}$ Antibiotics can be considered a therapeutic alternative to non-absorbable disaccharides in acute hepatic encephalopathy but in chronic encephalopathy should be reserved for patients who respond poorly to non-absorbable disaccharides. ${ }^{12}$

We performed a systematic review to assess the beneficial and harmful effects of non-absorbable disaccharides for hepatic encephalopathy and to compare them with antibiotics.

\section{Methods}

The review was performed according to a published protocol $^{13}$ and reported according to the QUOROM statement. ${ }^{14}$

\section{Searching}

We searched the Cochrane Hepato-Biliary Group controlled trials register, the Cochrane Library, Medline, and Embase up to March 2003. Included terms were "hepatic encephalopathy or cirrhosis", and "lactulose, lactitol, or disaccharide", and "random* or clinical". ${ }^{13}$ We screened bibliographies of relevant articles and conference proceedings and wrote to experts and pharmaceutical companies.

Selection-We included all randomised trials that compared non-absorbable disaccharides (lactulose and lactitol) with placebo, no treatment, or antibiotics for hepatic encephalopathy. Inclusion was regardless of publication status, language, or blinding. Included patients had acute, chronic, or minimal hepatic encephalopathy.

Validity assessment-Two reviewers independently assessed trial quality ${ }^{15}{ }^{16}$ by examining three components: generation of allocation sequence (classified as adequate if based on computer generated random numbers, tables of random numbers, or similar), concealment of allocation (classified as adequate if based on central randomisation, sealed envelopes, or similar), and blinding (classified as adequate if the trial was described as double blind or had blinded outcome assessment). ${ }^{13}$ We classified trials with adequate concealment of allocation and adequate blinding as high quality.

Data abstraction - Two reviewers (BA-N and LLG) independently extracted data from each trial. Our primary outcome measures were the numbers of patients without improvement of hepatic encephalopathy and all cause mortality. Improvement was defined as partial or complete resolution of clinical or 


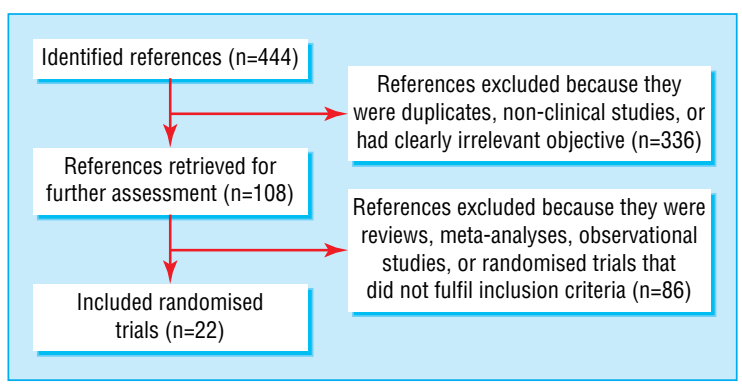

Fig 1 Selection process of eligible randomised trials from all identified references

subclinical symptoms of hepatic encephalopathy. Secondary outcome measures were adverse events, number connection test result, and blood ammonia concentration. In the number connection test, participants are instructed to connect numbers printed on a page consecutively from 1 to 25 as quickly as possible. The test score is the time the patient needs to perform the test, including the time needed to correct any errors. A low score represents a good performance. All outcomes were assessed at the end of treatment and maximum follow up.

Trial characteristics - We extracted the type and cause of the underlying liver disease, type of hepatic encephalopathy (acute, chronic, or minimal); mean age; proportion of men; number of patients randomised to each intervention arm; type, dose, and duration of treatment; mode of administration; trial quality ${ }^{15}$; trial design (parallel or crossover); duration of follow up; and number of dropouts. We sought data on all patients, irrespective of compliance or follow up. Primary investigators were contacted if data were missing.

Quantitative data synthesis-All data were analysed on the basis of intention to treat, including all randomised patients irrespective of compliance or follow up. If patients had missing outcome data, we carried forward the last reported observed response. ${ }^{17}$ Data from the first period of crossover trials were included. Binary outcomes were expressed as relative risks with 95\% confidence intervals. Continuous outcomes were expressed as weighted mean difference with $95 \%$ confidence intervals. We used a random effects mode ${ }^{18}$ because we anticipated clinical variability between trials. Statistical heterogeneity was explored by the $\chi^{2}$ test with significance set at $\mathrm{P}<0.1$. Potential sources of heterogeneity were explored through subgroup analyses with regard to the quality of methods and type of hepatic encephalopathy. We used the test of interaction ${ }^{19}$ to compare the difference between the estimates of subgroup analyses. Analyses were performed in Review Manager version 4.2.2. for Windows and SPSS version 11.0 for Windows.

\section{Results}

Figure 1 summarises the literature search. We included 22 trials that assessed lactulose or lactitol versus placebo, no treatment, or antibiotics. ${ }^{56}{ }^{20-39}$ Two trials were published as abstracts. ${ }^{32}{ }^{37}$ The remaining were published as full articles. Eighteen trials used a parallel group design and four a crossover design. All trials were described as randomised, but adequate generation of the allocation sequence was described in only four. ${ }^{22} 303139$ Treatment allocation was adequately concealed in 10 trials, ${ }^{5} 620-2326303639$ double blinding was reported in 15 trials, ${ }^{5} 620-252732-34363839$ and one trial had blinded outcome assessment. ${ }^{30}$ We classified nine trials as high quality. ${ }^{5}{ }^{5} 20-23303639$

\section{Lactulose or lactitol $v$ placebo or no intervention}

Ten trials with 280 patients ( $75 \%$ men) assessed lactulose or lactitol versus placebo or no intervention (table 1). ${ }^{20-29}$ All patients had cirrhosis and acute, ${ }^{25}$ chronic, ${ }^{20}{ }^{22-24}$ acute or chronic, ${ }^{21}$ or minimal hepatic encephalopathy. ${ }^{26-29}$ Eight trials assessed oral lactulose, ${ }^{20-24} 262829$ one assessed oral lactitol, ${ }^{27}$ and one assessed lactitol enemas. ${ }^{25}$ The daily mean doses of lactulose ranged from $30 \mathrm{~g}$ to $84 \mathrm{~g}$ (median $50 \mathrm{~g}$ ). In six trials the dose was adjusted to obtain two to three semisoft stools per day. The median duration of treatment was 15 days (range 5 to 360 days). None of the trials followed up patients after the end of treatment.

Trial results were homogeneous. Compared with placebo or no intervention, lactulose and lactitol seemed to reduce the risk of no improvement of hepatic encephalopathy (relative risk 0.62, $95 \%$ confidence interval 0.46 to 0.84 , six trials; fig 2 ). This result was not robust when trials were stratified by quality. High quality trials found no significant effect of lactulose or lactitol on the risk of no improvement $(0.92,0.42$ to 2.04 , two trials; fig 2$)$, whereas low quality trials found a significant beneficial effect of lactulose or lactitol $(0.57,0.40$ to 0.83 , four trials; fig 2$)$. Although this difference in treatment response was not significant $(\mathrm{P}=0.3$ by test of interaction), it is noteworthy that the event rate in the control groups was significantly associated with quality of methods (high quality trials $38 \%$, low quality trials $78 \% ; \mathrm{P}=0.0005$ with $\chi^{2}$ test). The event rate in the experimental group was not significantly different in trials with high (35\%) and low (43\%) quality $(\mathrm{P}=0.5$ with $\chi^{2}$ test). The treatment responses in acute, chronic, and minimal hepatic encephalopathy did not differ significantly. However, there was no significant effect of lactulose or lactitol on

Table 1 Randomised trials of non-absorbable disaccharides versus placebo or no intervention in treatment of patients with hepatic encephalopathy

\begin{tabular}{|c|c|c|c|c|c|c|c|c|c|}
\hline & \multirow[b]{2}{*}{ Study design } & \multirow[b]{2}{*}{ Quality* } & \multirow{2}{*}{$\begin{array}{l}\text { No of patients } \\
\text { randomised }\end{array}$} & \multirow{2}{*}{$\begin{array}{l}\text { Type of hepatic } \\
\text { encephalopathy }\end{array}$} & \multirow{2}{*}{$\begin{array}{l}\text { Experimental/control } \\
\text { intervention }\end{array}$} & \multicolumn{2}{|c|}{$\begin{array}{l}\text { No of patients without } \\
\text { improvement/total† }\end{array}$} & \multicolumn{2}{|c|}{ No of dropouts/total } \\
\hline & & & & & & Experimental & Control & Experimental & Control \\
\hline Elkington $1969^{20}$ & Crossover & High & 7 & Chronic & Lactulose/sorbitol & \multicolumn{2}{|l|}{$\ddagger$} & \multicolumn{2}{|c|}{ Not described } \\
\hline Simmons $1970^{21}$ & Parallel & High & 26 & Acute + chronic & Lactulose/glucose & $4 / 14$ & $5 / 12$ & $3 / 14$ & $2 / 12$ \\
\hline Rodgers $1973^{22}$ & Crossover & High & 6 & Chronic & Lactulose/sorbitol & \multicolumn{2}{|l|}{$\ddagger$} & \multicolumn{2}{|l|}{3} \\
\hline Germain $1973^{23}$ & Parallel & High & 18 & Chronic & Lactulose/saccharose & $4 / 9$ & $3 / 9$ & Non & \\
\hline Corazza $1982^{24}$ & Parallel & Low & 32 & Chronic & Lactulose/placebo & \multicolumn{2}{|l|}{$\S$} & \multicolumn{2}{|c|}{ Unknown } \\
\hline Uribe $1987^{25}$ & Parallel & Low & 15 & Acute & $\begin{array}{l}\text { Lactitol enemas/tap water } \\
\text { enemas }\end{array}$ & $0 / 10$ & $4 / 5$ & \multicolumn{2}{|c|}{ Unknown } \\
\hline Watanabe $1997^{26}$ & Parallel & Low & 36 & Minimal & Lactulose/no treatment & $12 / 22$ & $11 / 14$ & $2 / 22$ & $1 / 14$ \\
\hline Shi $1997^{27}$ & Parallel & Low & 31 & Minimal & Lactitol/glucose & \multicolumn{2}{|l|}{$\S$} & \multicolumn{2}{|c|}{ Unknown } \\
\hline Li $1999^{28}$ & Parallel & Low & 86 & Minimal & Lactulose/no treatment & $22 / 48$ & $27 / 38$ & \multicolumn{2}{|c|}{ Unknown } \\
\hline Dhiman $2000^{29}$ & Parallel & Low & 26 & Minimal & Lactulose/no treatment & $6 / 14$ & $12 / 12$ & $4 / 14$ & $4 / 12$ \\
\hline
\end{tabular}

*Classified with adequate allocation concealment and adequate blinding as high quality.

†Improvement defined as partial or complete resolution of clinical or subclinical symptoms of hepatic encephalopathy.

fLactulose and sorbitol reported to be equally effective, but numerical data not available.

§Lactulose/lactitol reported to be superior to placebo, but numerical data not available. 


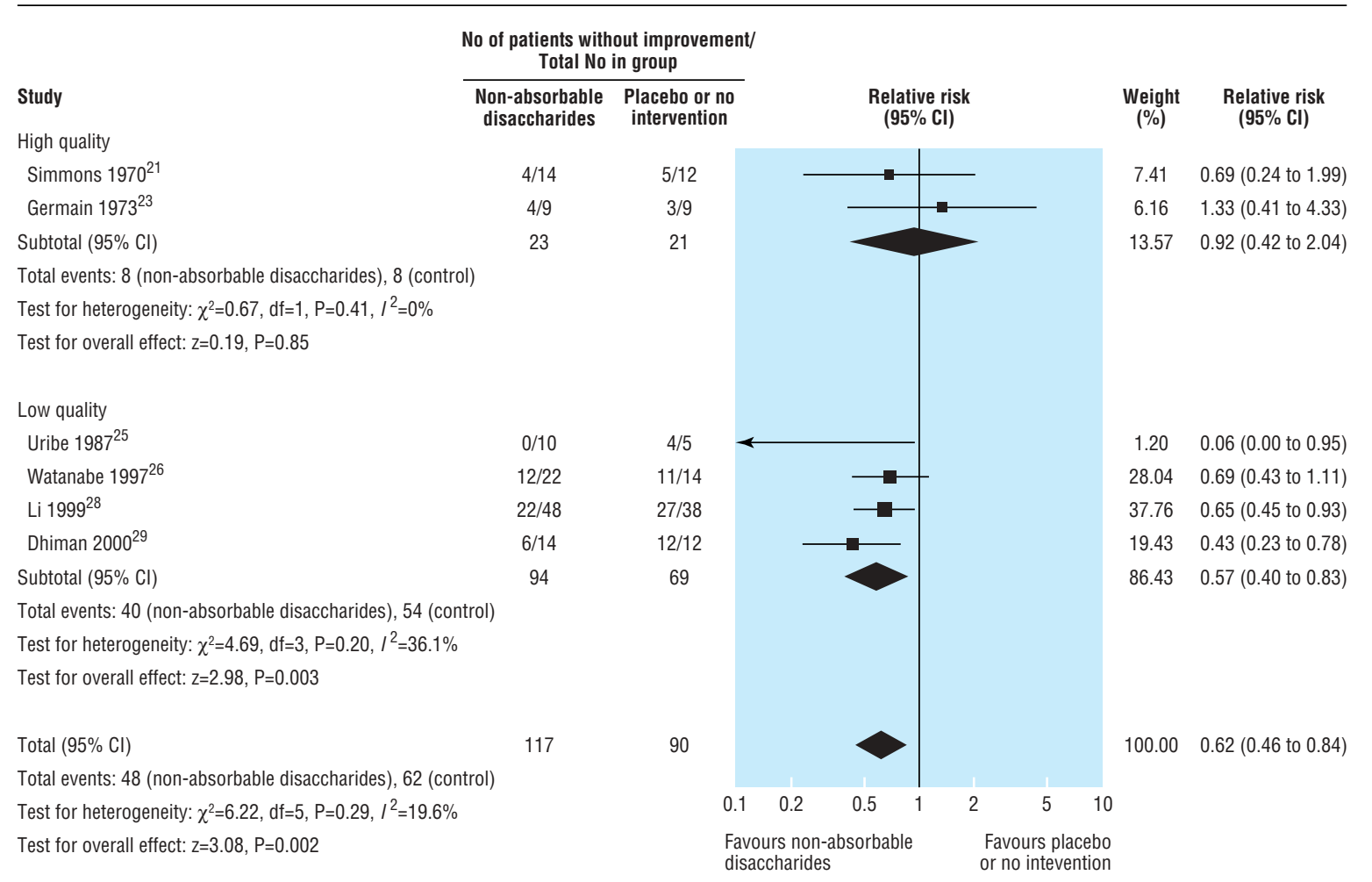

Fig 2 Number of patients without improvement of hepatic encephalopathy in trials on non-absorbable disaccharides versus placebo or no intervention, stratified according to quality of methods

acute $(0.27,0.02$ to 3.28 , two trials) or chronic hepatic encephalopathy (1.33, 0.41 to 4.33 , one trial). Trials in patients with minimal hepatic encephalopathy found that lactulose or lactitol significantly reduced the risk of no improvement assessed by various psychometric tests $(0.61,0.47$ to 0.79 , three trials). These trials were all of low methodological quality.

Compared with placebo or no intervention, lactulose and lactitol had no significant effect on mortality $(0.41,0.02$ to 8.68 , four trials) or the number connection test result (weighted mean difference -9.0 seconds, -20.1 to 2.1 , one trial) but tended to lower blood ammonia $(-8.16 \mu \mathrm{mol} / \mathrm{l},-16.44 \mu \mathrm{mol} / \mathrm{l}$ to 0.18 $\mu \mathrm{mol} / \mathrm{l}$, four trials). Data on adverse events were incompletely reported. Most trials mentioned adverse events associated only with non-absorbable disaccharides. We were therefore unable to perform a reliable meta-analysis of this outcome. None of the reported adverse events were serious, and all originated from the gastrointestinal tract (diarrhoea, flatulence, abdominal pain, or nausea).

\section{Lactulose or lactitol versus antibiotics}

Twelve trials with 698 patients (72\% men) assessed lactulose or lactitol versus antibiotics (table 2). ${ }^{5} 6$ 30-39 All patients had cirrhosis and acute, ${ }^{632} 39$ chronic, ${ }^{531353638}$ acute or chronic, ${ }^{30}$ or presumed chronic hepatic encephalopathy. 3434 Nine trials assessed oral lactulose, ${ }^{56303133-37}$ and three trials assessed oral lactitol. $^{32}{ }^{38}{ }^{39}$ The daily mean dose of lactulose ranged from $30 \mathrm{~g}$ to $120 \mathrm{~g}$ (median $59 \mathrm{~g}$ ) and of lactitol from $30 \mathrm{~g}$ to $60 \mathrm{~g}$ (median $60 \mathrm{~g})$. The antibiotics were neomycin, ${ }^{5}{ }^{60}$ ribostamycin, ${ }^{31}$ vancomycin,${ }^{32}$ or rifaximin. ${ }^{33-39}$ The median duration of treatment was 15 days (range 5-90 days). One trial assessed all outcomes 15 days after the end of treatment, ${ }^{38}$ and one reported mortality 28 days after the end of treatment. ${ }^{39}$ All other trials followed the patients only to the end of treatment.

Trial results were homogeneous. Compared with antibiotics, patients taking lactulose or lactitol had a significantly higher risk of no improvement of hepatic encephalopathy $(1.24,1.02$ to 1.50, 10 trials; fig 3). We found no significant difference in response to treatment between aminoglycosides and rifaximin $(\mathrm{P}=0.2$ by test of interaction) or when trials were stratified by quality or type of hepatic encephalopathy. We found no significantly different effect on mortality between nonabsorbable disaccharides and antibiotics $(0.90,0.48$ to 1.67 , five trials) or on adverse events (1.62, 0.57 to 4.58 , eight trials). None of the reported adverse events were serious, and all originated from the gastrointestinal tract (diarrhoea, flatulence, abdominal pain, or nausea). Compared with antibiotics, patients on lactulose or lactitol took on average six more seconds to complete the number connection test (weighted mean difference 6.4 seconds, 1.4 seconds to 11.3 seconds, six trials) and had higher blood ammonia concentrations $(2.35 \mu \mathrm{mol} / \mathrm{l}, 0.06 \mu \mathrm{mol} / \mathrm{l}$ to 4.64 $\mu \mathrm{mol} / \mathrm{l}, 10$ trials).

\section{Discussion}

We did not find sufficient evidence to determine whether lactulose or lactitol have a significant beneficial effect on patients with hepatic encephalopathy. In our overall analysis non-absorbable disaccharides seemed to improve encephalopathy, but this effect was seen in only low quality trials.

The beneficial effect in low quality trials was related to significantly worse rates of improvement in the control group. This finding concurs with empirical evidence showing that low quality trials exaggerate the beneficial effects of treatment. ${ }^{15} 1640$ Accordingly, the overall result may reflect bias because of the low methodological quality of most of the included trials. Our results may also be inflated by publication bias.

We found no significant effect of non-absorbable disaccharides on acute or chronic hepatic encephalopathy. Only low quality trials in patients with minimal hepatic encephalopathy found that lactulose had a beneficial effect, as assessed by various 
Table 2 Randomised trials on non-absorbable disaccharides versus antibiotics in treatment of patients with hepatic encephalopathy

\begin{tabular}{|c|c|c|c|c|c|c|c|c|c|}
\hline & \multirow[b]{2}{*}{ Study design } & \multirow[b]{2}{*}{ Quality* } & \multirow{2}{*}{$\begin{array}{c}\text { No of } \\
\text { patients } \\
\text { randomised }\end{array}$} & \multirow{2}{*}{$\begin{array}{l}\text { Type of hepatic } \\
\text { encephalopathy }\end{array}$} & \multirow{2}{*}{$\begin{array}{l}\text { Experimental/control } \\
\text { intervention }\end{array}$} & \multicolumn{2}{|c|}{$\begin{array}{l}\text { No of patients without } \\
\text { improvement/total† }\end{array}$} & \multicolumn{2}{|c|}{ No of dropouts/total } \\
\hline & & & & & & Experimental & Antibiotics & Experimental & Antibiotics \\
\hline Conn $1977^{5}$ & Crossover & High & 33 & Chronic & $\begin{array}{l}\text { Lactulose + } \\
\text { placebo/neomycin + } \\
\text { sorbitol }\end{array}$ & $3 / 18$ & $2 / 15$ & \multicolumn{2}{|c|}{ None in 1st period } \\
\hline Atterbury $1978^{6}$ & Parallel & High & 47 & Acute & $\begin{array}{l}\text { Lactulose + } \\
\text { placebo/neomycin + } \\
\text { sorbitol }\end{array}$ & $4 / 23$ & $4 / 24$ & $1 / 23$ & $1 / 24$ \\
\hline Orlandi $1981^{30}$ & Parallel & High & 190 & Acute + chronic & $\begin{array}{l}\text { Lactulose/neomycin + } \\
\text { magnesium sulfate }\end{array}$ & $63 / 91$ & $48 / 82$ & & $17 \S$ \\
\hline Russo $1989^{31}$ & Crossover & Low & 15 & Chronic & Lactulose/ribostamycin & $1 / 8$ & $2 / 7$ & \multicolumn{2}{|c|}{ Unknown } \\
\hline Blanc $1993^{32}$ & Parallel & Low & 60 & Acute & Lactitol/vancomycin & $9 / 29$ & $10 / 31$ & $2 / 29$ & $2 / 31$ \\
\hline Bucci $1993^{33}$ & Parallel & Low & 58 & Unknown & $\begin{array}{l}\text { Lactulose + } \\
\text { placebo/rifaximin + sorbitol }\end{array}$ & \multicolumn{2}{|c|}{$\ddagger$} & \multicolumn{2}{|c|}{ Unknown } \\
\hline Fera $1993^{34}$ & Parallel & Low & 40 & Unknown & $\begin{array}{l}\text { Lactulose }+ \\
\text { placebo/rifaximin }+ \\
\text { placebo }\end{array}$ & $4 / 20$ & $0 / 20$ & \multicolumn{2}{|c|}{ Unknown } \\
\hline Festi $1993^{35}$ & Parallel & Low & 21 & Chronic & Lactulose/rifaximin & \multicolumn{2}{|c|}{$\ddagger$} & \multicolumn{2}{|c|}{ Unknown } \\
\hline Massa $1993^{36}$ & Parallel & High & 40 & Chronic & $\begin{array}{l}\text { Lactulose + } \\
\text { placebo/rifaximin + sorbitol }\end{array}$ & $2 / 20$ & $0 / 20$ & \multicolumn{2}{|c|}{ Unknown } \\
\hline Song $2000^{37}$ & Parallel & Low & 64 & Unknown & Lactulose/rifaximin & $7 / 25$ & $8 / 39$ & $1 / 25$ & $1 / 39$ \\
\hline Loguercio $2003^{38}$ & Parallel & Low & 27 & Chronic & $\begin{array}{l}\text { Lactitol + placebo/rifaximin } \\
+ \text { placebo }\end{array}$ & $11 / 13$ & $6 / 14$ & $3 / 13$ & $2 / 14$ \\
\hline Mas $2003^{39}$ & Parallel & High & 103 & Acute & $\begin{array}{l}\text { Lactitol + placebo/rifaximin } \\
+ \text { placebo }\end{array}$ & $12 / 53$ & $10 / 50$ & $7 / 53$ & $8 / 50$ \\
\hline
\end{tabular}

*Classified with adequate allocation concealment and adequate blinding as high quality.

†Improvement defined as partial or complete resolution of clinical or subclinical symptoms of hepatic encephalopathy.

tExperimental and control intervention reported to be equally effective but numerical data not available.

$\S$ Exact number of dropouts in each intervention group not reported and accordingly it was not possible to perform intention to treat analysis for this trial.

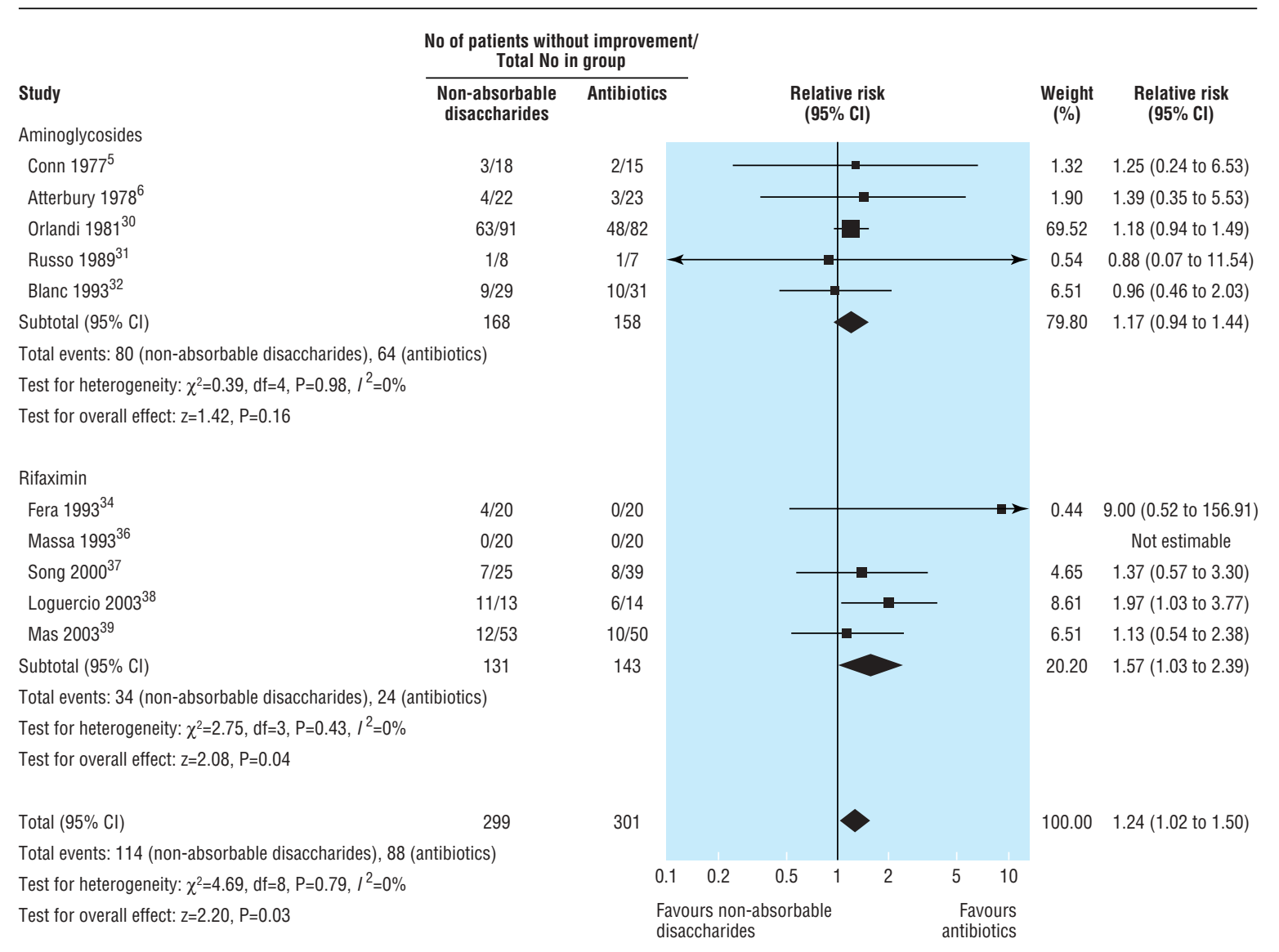

Fig 3 Number of patients without improvement of hepatic encephalopathy in trials on non-absorbable disaccharides versus antibiotics, stratified according to type of antibiotic 
non-validated psychometric tests. The clinical relevance of these tests is uncertain. ${ }^{41}$

Lactulose has been used as the standard treatment for hepatic encephalopathy, and its efficacy has been considered to be beyond doubt. ${ }^{27242542}$ However, when it was introduced, the few trials that compared lactulose against placebo found no beneficial effect of lactulose. ${ }^{21}{ }^{23}$ It was implemented in clinical practice because two trials found it "equally effective" to neomycin, ${ }^{56}$ which had been the standard treatment for hepatic encephalopathy since $1957 .^{43}$ There are two major pitfalls in this reasoning. Firstly, the efficacy of neomycin in hepatic encephalopathy has never been shown. We identified only one randomised trial that compared neomycin with placebo ${ }^{44}$ and one that compared neomycin plus lactulose with placebo, ${ }^{45}$ both for acute hepatic encephalopathy. Both trials found no significant beneficial effects of neomycin. Secondly, lactulose was considered as equally effective to neomycin because event rates in intervention groups were not significantly different. ${ }^{5}{ }^{6}$ However, lack of statistical significance does not imply that treatments have equal effects. ${ }^{46}$ Both trials were small,,${ }^{56}$ and neither reported sample size calculations based on an equivalence hypothesis or stated a margin of equivalence. ${ }^{46}{ }^{47}$ It would require a far larger sample size than these two trials (a total of 78 patients) to establish with confidence that lactulose and neomycin have comparable effects.

Later on, new trials compared other antibiotics to non-absorbable disaccharides for hepatic encephalopathy. None was set up as an equivalence trial. Sample size calculations with statements implying an equivalence hypothesis or a margin of equivalence were not reported in any of the trials. All were underpowered to show equivalence. Nevertheless, all trials concluded equivalence from the lack of statistical significance. ${ }^{30-39}$ It seems that the research was continuously building up on both insufficient evidence and inadequate methods. Our analyses indicate that antibiotics are statistically superior to non-absorbable disaccharides in improving hepatic encephalopathy and lowering blood ammonia concentrations. However, it is unclear whether the effects are clinically important. Considering this, the lack of effect of antibiotics in placebo controlled trials, ${ }^{44}{ }^{45}$ the risk of multiresistance, ${ }^{48}$ and the potential risk of severe adverse events ${ }^{5}$ lead us to conclude that there is insufficient evidence to recommend the use of antibiotics for hepatic encephalopathy.

\section{Mechanisms}

When assessing intervention effects for hepatic encephalopathy, it is important to consider the fluctuating course as well as the impact of treating precipitating factors in acute hepatic encephalopathy. Well conducted placebo controlled trials on the use of ornithine aspartate in patients with minimal or chronic hepatic encephalopathy ${ }^{49}{ }^{50}$ and lactulose plus neomycin ${ }^{45}$ in those with acute hepatic encephalopathy found improvement rates in the placebo group ranging from $40 \%$ to $70 \%$. Many clinicians claim to have witnessed beneficial effects of nonabsorbable disaccharides on patients with hepatic encephalopathy. This effect may represent a high rate of spontaneous improvement and successful treatment of precipitating factors.

\section{Implications}

Non-absorbable disaccharides seem to have been introduced into clinical practice without appropriate documentation. This leads to at least three major problems. Firstly, patients are given a treatment of uncertain efficacy. It might be beneficial; it might be unfavourable. Secondly, there is reluctance towards performing randomised trials to assess lactulose or lactitol versus placebo because it is considered unethical. Thirdly, most randomised

\section{What is already known on this topic}

Non-absorbable disaccharides are considered standard treatment for hepatic encephalopathy

Non-absorbable disaccharides serve as control treatment in most trials of new drugs for hepatic encephalopathy

What this study adds

There is insufficient evidence to determine whether non-absorbable disaccharides are of benefit to patients with hepatic encephalopathy

Antibiotics seem superior to non-absorbable disaccharides in improving hepatic encephalopathy, but it is unclear whether this difference is clinically important

Non-absorbable disaccharides should not be used as the comparator in randomised trials on hepatic encephalopathy

trials on new treatments for hepatic encephalopathy use lactulose as comparator. New treatments are considered effective if improvement rates do not differ significantly from the group treated with lactulose, although trials are vastly underpowered to show equivalence. This approach is most problematic. Nonabsorbable disaccharides should not serve as comparator in randomised trials on hepatic encephalopathy until other trials have shown that lactulose or lactitol has any beneficial effect on hepatic encephalopathy.

We thank the patients who took part in the reviewed trials; the researchers who provided us with additional information; Iørgen Hilden, department of biostatistics, University of Copenhagen, for statistical support; and Peter Gøtzsche, Nordic Cochrane Centre, for valuable comments on an earlier draft of this review. This review is an abbreviated version of a Cochrane systematic review. The full version will be published in the Cochrane Library 2004, Issue 2. Cochrane Reviews are regularly updated as new evidence emerges and in response to comments and criticisms, and the Cochrane Library should be consulted for the most recent version of the review.

Contributors: BA-N drafted the protocol and paper, performed the literature searches, identified trials, extracted data, and performed the statistical analyses. LLG identified trials and extracted data. All reviewers contributed to the writing of the protocol and review and all have approved of the final version. BA-N is guarantor.

Funding: Danish Centre for Evaluation and Health Technology Assessment (DACEHTA), Danish Medical Research Council, and Copenhagen Hospital Corporation's Medical Research Council.

Competing interests: None declared.

Ethical approval: Not required.

1 Gitlin N. Hepatic encephalopathy In: Zakim D, Boyer TD, eds. Hepatology. A textbook of liver disease. Philadelphia: WB Saunders, 1996:605-17.

2 Conn HO, Lieberthal MM. Lactulose in the management of chronic portal-systemic encephalopathy. In: Conn HO, Lieberthal MM, eds. The hepatic coma syndromes and lactulose. Baltimore: Williams \& Wilkins, 1979:323-39.

3 Bircher J, Muller J, Guggenheim P, Hammerli UP. Treatment of chronic portal-systemic encephalopathy with lactulose. Lancet 1966;i:890-2.

4 Weissenborn K. Recent developments in the pathophysiology and treatment of hepatic encephalopathy. Baillieres Clin Gastroenterol 1992;6:609-30.

5 Conn HO, Leevy CM, Vlacevic ZR, Rodgers JB, Maddrey WC, Seef L. Comparison of lactulose and neomycin in the treatment of chronic portal-systemic encephalopathy. A double blind controlled trial. Gastroenterology 1977;72:573-83.

6 Atterbury CE, Maddrey WC, Conn HO. Neomycin-sorbitol and lactulose in the treatment of acute portal-systemic encephalopathy. A controlled, double-blind clinical trial. Am J Dig Dis 1978;23:398-406.

7 Morgan MY, Hawley KE. Lactitol vs. lactulose in the treatment of acute hepatic encephalopathy in cirrhotic patients: a double-blind, randomized trial. Hepatology 1987;7:1278-84.

8 Morgan MY, Hawley KE, Stambuk D. Lactitol versus lactulose in the treatment of chronic hepatic encephalopathy. A double-blind, randomised, cross-over study. J Hepatol 1987;4:236-44. 
9 Blanc P, Daures JP, Rouillon JM, Peray P, Pierrugues R, Larrey D. Lactitol or lactulose in the treatment of chronic hepatic encephalopathy: results of a meta-analysis. Hepatology 1992;15:222-8.

10 Camma C, Fiorello F, Tine F, Marchesini G, Fabbri A, Pagliaro L. Lactitol in treatment of chronic encephalopathy. A meta-analysis. Dig Dis Sci 1993;38:916-22.

11 Morgan MY. Nutritional aspects of liver and biliary disease. In: Bircher J, Benhamou JP, McIntyre N, Rizzetto M, Rodes J, eds. Oxford textbook of clinical hepatology. Oxford: Oxford University Press, 1999:1923-81.

12 Blei AT, Cordoba J. Hepatic encephalopathy. Am J Gastroenterol 2001;96:1968-76.

13 Als-Nielsen B, Kjaergard LL, Gluud C. Nonabsorbable disaccharides for hepatic encephalopathy (protocol for a Cochrane review). In: Cochrane Library, Issue 2. Oxford: Update Software, 2001.

14 Moher D, Cook DJ, Eastwood S, Olkin I, Rennie D, Stroup DF. Improving the quality of reports of meta-analyses of randomised controlled trials: the QUOROM statement. Lancet 1999;354:1896-900.

15 Schulz KF, Chalmers I, Hayes RJ, Altman DG. Empirical evidence of bias. Dimensions of methodological quality associated with estimates of treatment effects in controlled trials. JAMA 1995;273:408-12.

16 Kjaergard LL, Villumsen J, Gluud C. Reported methodological quality and discrepancies between small and large randomized trials in meta-analyses. Ann Intern Med 2001;135:982-9.

17 Hollis S, Campbell F. What is meant by intention to treat analysis? Survey of published randomised controlled trials. BMJ 1999;319:670-4.

18 DerSimonian R, Laird N. Meta-analysis in clinical trials. Control Clin Trials 1986;7:17788.

19 Altman DG, Bland JM. Interaction revisited: the difference between two estimates. BMJ 2003;326:219.

20 Elkington SG, Floch MH, Conn HO. Lactulose in the treatment of chronic portal-systemic encephalopathy. A double-blind clinical trial. $N$ Engl J Med 1969;281:408-12.

21 Simmons F, Goldstein H, Boyle JD. A controlled clinical trial of lactulose in hepatic encephalopathy. Gastroenterology 1970;59:827-32.

22 Rodgers JB Jr, Kiley JE, Balint JA. Comparison of results of long-term treatment of chronic hepatic encephalopathy with lactulose and sorbitol. Am J Gastroenterol 1973;60:459-65.

23 Germain L, Frexinos J, Louis A, Ribet A. Double blind study of lactulose in 18 patients with chronic hepatic encephalopathy after portocaval shunt. Arch Fr Mal App Dig 1973;62:293-302

24 Corazza GR, Tacconi C, Zoli G, Somarolli M, D'Ambro A, Bernardi M. Use of pyridoxine-alpha-ketoglutarate (PAK) in hepatic encephalopathy. Int J Clin Pharmacol Res 1982;2:7-13.

25 Uribe M, Campollo O, Vargas F, Ravelli GP, Mundo F, Zapata L. Acidifying enemas (lactitol and lactose) vs. nonacidifying enemas (tap water) to treat acute portal-systemic encephalopathy: a double-blind, randomized clinical trial. Hepatology 1987;7:639-43.

26 Watanabe A, Sakai T, Sato S, Imai F, Ohto M, Arakawa Y. Clinical efficacy of lactulose in cirrhotic patients with and without subclinical hepatic encephalopathy. Hepatology 1997;26:1410-4.

27 Shi H, Liu HY, Fu Z, Zhu L, Chen WZ. Lactitol in treatment of subclinical hepatic encephalopathy: a double blind placebo-controlled randomised trial [in Chinese]. Chinese J Digestion 1997;17:221-3.

$28 \mathrm{Li}$, Zhang H, Hong Y. Clinical effect of lactulose in the treatment of subclinical hepatic encephalopathy. Zhongguo Zhong Xi Yi Jie He Za Zhi 1999;9:13-5.

29 Dhiman RK, Sawhney MS, Chawla YK, Das G, Ram S, Dilawari JB. Efficacy of lactulose in cirrhotic patients with subclinical hepatic encephalopathy. Dig Dis Sci 2000;45:154952.

30 Orlandi F, Freddara U, Candelaresi MT, Morettini A, Corazza GR, Di Simone A. Comparison between neomycin and lactulose in 173 patients with hepatic encephalopathy: a randomized clinical study. Dig Dis Sci 1981;26:498-506.

31 Russo M, Galanti B, Nardiello S, Pizzella T, Ronga C, Giusti G. Ribostamycin for the treatment of hepatic encephalopathy: a crossover study with lactulose. Curr Ther Res Clin Exp 1989;45:133-41.
32 Blanc P, Couderc M, Peray P, Liautard J, Larrey D, Michel H, et al. Lactitol versus vancomycin in the treatment of acute hepatic encephalopathy: a double blind, randomized trial [abstract]. Gut 1993;34:46.

33 Bucci L, Palmieri GC. Double-blind, double-dummy comparison between treatment with rifaximin and lactulose in patients with medium to severe degree hepatic encephalopathy. Curr Med Res Opin 1993;13:109-18.

34 Fera G, Agostinacchio F, Nigro M, Schiraldi O, Ferrieri A. Rifaximin in the treatment of hepatic encephalopathy. Rev Eur Etud Clin Biol 1993;4:57-66.

35 Festi D, Mazzella G, Orsini M, Sottili S, Sangermano A, Li B. Rifaximin in the treatment of chronic hepatic encephalopathy; results of a multicenter study of efficacy and safety. Curr Ther Res Clin Exp 1993;54:598-609.

36 Massa P, Vallerino E, Dodero M. Treatment of hepatic encephalopathy with rifaximin: double-blind, double dummy study versus lactulose. Eur J Clin Res 1993;4:7-18.

37 Song H, Lee KS, Kim MH, Paik YH, Moon BS, Yoon SH. The clinical efficacy of rifaximin in the treatment of hepatic encephalopathy (comparison with lactulose) [abstract]. Hepatology 2000;32:407.

38 Loguercio C, Federico A, De Girolamo V, Ferrieri A, Del Vicchio BD. Cyclic treatment of chronic hepatic encephalopathy with rifaximin. Results of a double-blind clinical study. Minerva Gastroenterol Dietol 2003:49:53-62.

39 Mas A, Rodes J, Sunyer L, Rodrigo L, Planas R, Vargas V. Comparison of rifaximin and lactitol in the treatment of acute hepatic encephalopathy: results of a randomized, double-blind, double-dummy, controlled clinical trial.J Hepatol 2003;38:51-8.

40 Jüni P, Altman D, Egger M. Systematic reviews in health care: assessing the quality of controlled clinical trials. BMJ 2001;323:42-6.

41 Weissenborn K. Minimal hepatic encephalopathy: a permanent source of discussion. Hepatology 2002;35:494-6.

42 Conn HO. A clinical hepatologist's predictions about non-absorbed carbohydrates for the early twenty-first century. Scand J Gastroenterol Suppl 1997;222:88-92.

43 Dawson AM, McLaren J, Sherlock S. Neomycin in the treatment of hepatic coma? Lancet 1957;273:1263-8.

44 Strauss E, Tramote R, Silva EP, Caly WR, Honain NZ, Maffei RA. Double-blind randomized clinical trial comparing neomycin and placebo in the treatment of exogenous hepatic encephalopathy. Hepatogastroenterology 1992:39:542-5.

45 Blanc P, Daures JP, Liautard J, Buttigieg R, Desprez D, Pageaux G. Lactulose-neomycin combination versus placebo in the treatment of acute hepatic encephalopathy. Results of a randomized controlled trial. Gastroenterol Clin Biol 1994;18:1063-8.

46 Pocock SJ. The size of a clinical trial. In: Clinical trials: a practical approach. Chichester: John Wiley, 1983:123-42.

47 Piaggio G, Pinol AP. Use of the equivalence approach in reproductive health clinical trials. Stat Med 2001;20:3571-7.

48 Hunter PA. Coping with the rising tide of resistance to antimicrobial agents. Drug News Perspect 2001:14:309-17.

49 Kircheis G, Nilius R, Held C, Berndt H, Buchner M, Gortelmeyer R. Therapeutic efficacy of L-ornithine-L-aspartate infusions in patients with cirrhosis and hepatic encephalopathy: results of a placebo-controlled, double-blind study. Hepatology 1997;25:1351-60.

50 Stauch S, Kircheis G, Adler G, Beckh K, Ditschuneit H, Gortelmeyer R. Oral L-ornithine-L-aspartate therapy of chronic hepatic encephalopathy: results of a placebo-controlled double-blind study.J Hepatol 1998;28:856-64.

(Accepted 20 February 2004)

doi 10.1136/bmj.38048.506134.EE

Cochrane Hepato-Biliary Group, Copenhagen Trial Unit, Centre for Clinical Intervention Research, Copenhagen University Hospital, Department 7102, H:S Rigshospitalet, DK-2100 Copenhagen, Denmark

Bodil Als-Nielsen research fellow

Lise L Gluud research fellow

Christian Gluud chief physician

Correspondence to: B Als-Nielsen bodil.a@ctu.rh.dk 